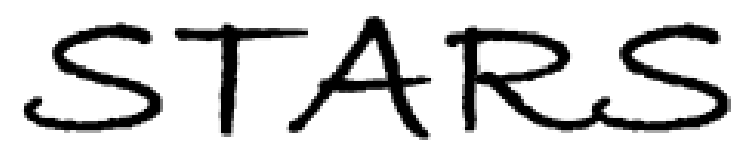

University of Central Florida

STARS

$1-1-2006$

\title{
Ultralow noise optical pulse generation in an actively mode-locked quantum-dot semiconductor laser
}

\author{
Myoung-Taek Choi \\ University of Central Florida \\ Ji-Myung Kim \\ University of Central Florida \\ Wangkuen Lee \\ University of Central Florida \\ Peter J. Delfyett \\ University of Central Florida
}

Find similar works at: https://stars.library.ucf.edu/facultybib2000

University of Central Florida Libraries http://library.ucf.edu

This Article is brought to you for free and open access by the Faculty Bibliography at STARS. It has been accepted for inclusion in Faculty Bibliography 2000s by an authorized administrator of STARS. For more information, please contactSTARS@ucf.edu.

\section{Recommended Citation}

Choi, Myoung-Taek; Kim, Ji-Myung; Lee, Wangkuen; and Delfyett, Peter J., "Ultralow noise optical pulse generation in an actively mode-locked quantum-dot semiconductor laser" (2006). Faculty Bibliography 2000s. 6027.

https://stars.library.ucf.edu/facultybib2000/6027

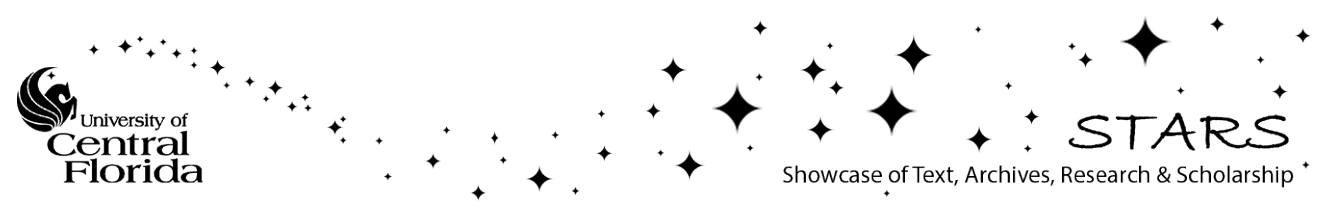




\section{Ultralow noise optical pulse generation in an actively mode-locked quantum-dot semiconductor laser}

Cite as: Appl. Phys. Lett. 88, 131106 (2006); https://doi.org/10.1063/1.2190443

Submitted: 26 December 2005 . Accepted: 13 February 2006 . Published Online: 28 March 2006

Myoung-Taek Choi, Ji-Myung Kim, Wangkuen Lee, and Peter J. Delfyett

\section{ARTICLES YOU MAY BE INTERESTED IN}

High-power picosecond and femtosecond pulse generation from a two-section mode-locked quantum-dot laser

Applied Physics Letters 87, 081107 (2005); https://doi.org/10.1063/1.2032608

Subpicosecond high-power mode locking using flared waveguide monolithic quantum-dot lasers

Applied Physics Letters 88, 133119 (2006); https://doi.org/10.1063/1.2186110

Stable mode locking via ground- or excited-state transitions in a two-section quantum-dot laser

Applied Physics Letters 89, 081124 (2006); https://doi.org/10.1063/1.2338767
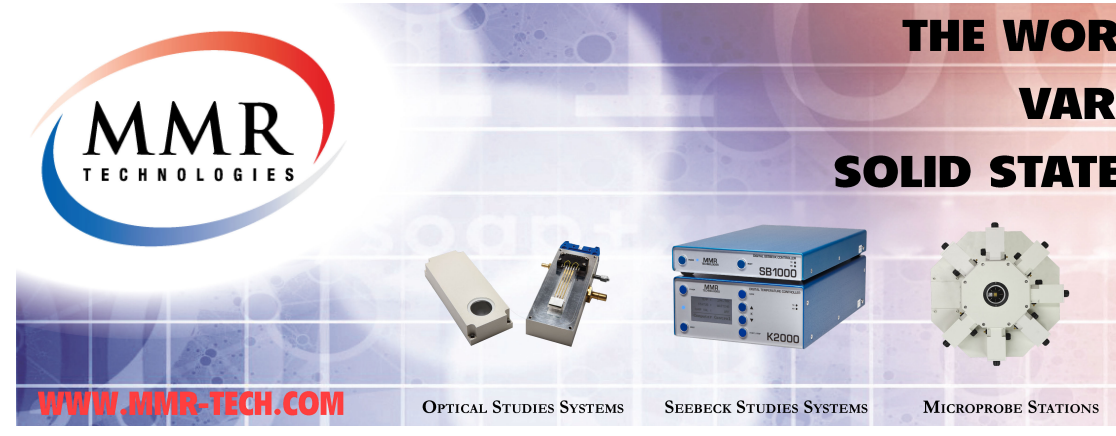

THE WORLD'S RESOURCE FOR VARIABLE TEMPERATURE 


\title{
Ultralow noise optical pulse generation in an actively mode-locked quantum-dot semiconductor laser
}

\author{
Myoung-Taek Choi, ${ }^{\text {a) }}$ Ji-Myung Kim, Wangkuen Lee, and Peter J. Delfyett ${ }^{\text {b) }}$ \\ College of Optics and Photonics/CREOL and FPCE, University of Central Florida, \\ 4000 Central Florida Boulevard, Orlando, Florida 32816
}

(Received 26 December 2005; accepted 13 February 2006; published online 28 March 2006)

\begin{abstract}
We report excellent noise performance of an external-cavity actively mode-locked laser based on quantum-dot gain medium. Optical pulse trains with less than $7.5 \mathrm{fs}$ residual timing jitter ( $1 \mathrm{~Hz}$ to $10 \mathrm{MHz}$ ) for a $12.8 \mathrm{GHz}$ harmonically mode-locked ring laser were obtained. This result represents, to our knowledge, the lowest residual jitter reported from actively mode-locked semiconductor lasers, and shows that quantum-dot mode-locked lasers are promising as sources of ultralow noise optical pulse trains. (C) 2006 American Institute of Physics.
\end{abstract}

[DOI: $10.1063 / 1.2190443$ ]

Low noise mode-locked lasers have important uses in high-bit-rate optical communications, optical sampling, optical clock distribution in optical interconnects, microwave signal generation, and precision metrology. Extensive research has been performed for developing and understanding quantum-well (QW) and bulk-based low noise mode-locked lasers. ${ }^{1-7}$ Recently, lasers and semiconductor optical amplifiers (SOAs) based on quantum-dot (QD) gain media have attracted much attention as next-generation light sources. The QD lasers have shown remarkable properties, such as temperature-insensitive operation, suppression of beam filamentation, reduced sensitivity to optical feedback, etc. Theoretical investigations show that QD SOAs may exhibit superior characteristics compared with conventional QW/bulkbased SOAs (Refs. 8 and 9) owing to the low loss, highly inverted population and ultrafast gain recovery time. High saturation output power, large gain, and lower noise figure have been verified experimentally. ${ }^{10}$

In this Letter, we investigate the performance of an actively mode-locked laser based on a QD SOA as a gain medium, for the first time to our knowledge. A unidirectional ring laser is employed for low noise optical pulse generation in order to avoid the spatial hole burning detrimental to wavelength stability in the SOA. ${ }^{11}$

The QD amplifier epistructure was grown with molecular beam epitaxy (MBE) on an $n+\mathrm{GaAs}$ substrate. The active region consists of ten layers of self-assembled InAs/GaAs quantum dots, covered with $5 \mathrm{~nm} \mathrm{In}_{0.15} \mathrm{Ga}_{0.85}$ As. SOAs with a $3.2 \mathrm{~mm}$ length and a $4 \mu \mathrm{m}$ ridge waveguide width were fabricated. The waveguide is tilted at an angle of $7^{\circ}$ relative to the cleaved facet to minimize the backreflection from the facet. Both facets were antireflection (AR) coated, and the device is mounted $p$ side up. The SOAs were kept at $20^{\circ} \mathrm{C}$ using a thermoelectric cooler control.

Figure 1 shows the schematic of the ring laser. The unidirectional ring laser was constructed using the QD SOA described above, a low loss Mach-Zender intensity modulator, an isolator, an output coupler, and polarization controllers. The cavity length was approximately $6 \mathrm{~m}$, corresponding to a fundamental cavity mode spacing of $25 \mathrm{MHz}$. The

\footnotetext{
${ }^{a}$ Electronic mail: mtchoi@creol.ucf.edu

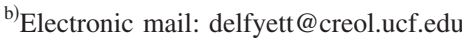

loss-modulated mode locking is achieved by dc biasing the SOA and applying the rf synthesizer signal to the high-speed intensity modulator, driven at $12.8 \mathrm{GHz}$. Active mode locking with low noise was well maintained over a broad range of dc bias conditions, ranging from 120 to $200 \mathrm{~mA}$. Mode locking was optimized for the lowest noise operation at a dc bias of $170 \mathrm{~mA}$ on the QD SOA, with an average output power of $\sim 1 \mathrm{~mW}$ from the oscillator. The laser output was amplified through another QD SOA to boost the optical power into the photodetector for further diagnosis. After amplification, the optical power was $\sim 6.2 \mathrm{~mW}$.

Figure 2(a) shows the optical spectrum of mode-locked pulses showing stable optical combs with more than $10 \mathrm{~dB}$ mode contrast, limited by the resolution of the spectrum analyzer. The number of combs contained in the full width at half maximum (FWHM) bandwidth of $2 \mathrm{~nm}(370 \mathrm{GHz})$ is 29 . The modulation of $\sim 0.3 \mathrm{~nm}$ period imposed on the spectrum is due to the polarization cross talk of the intensity modulator. The mode-locked pulse trains were detected by a high-speed photodetector (50 GHz bandwidth) and are shown in Fig. 2(b). The pulse width ranges from 24 to 28 ps in the low noise operating regime. The time bandwidth product (TBP) is $\sim 10$, implying that the pulses are highly chirped.

Figure 3(a) shows the rf spectrum near the carrier frequency of $12.8 \mathrm{GHz}$. The supermode ${ }^{12}$ spurs at offset frequencies of multiples of the fundamental cavity frequency $\sim 25 \mathrm{MHz}$ are seen around the prominent carrier. The effect

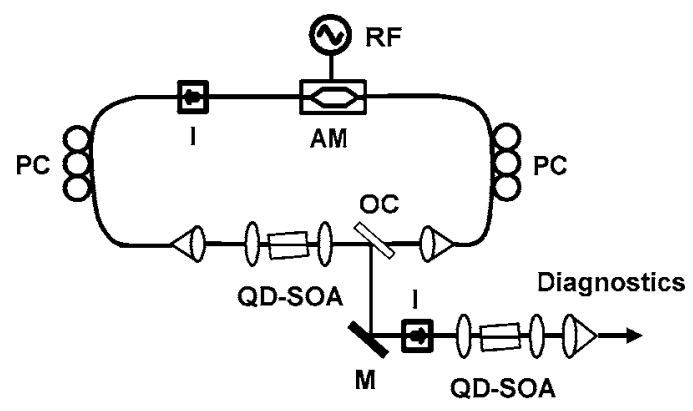

FIG. 1. Schematic of the unidirectional QD ring laser. QD SOA: quantumdot semiconductor optical amplifier, I: isolator, AM: amplitude modulator, OC: $20 \%$ output coupler, PC: polarization controller, rf: rf driving signal, and $\mathrm{M}$ : mirror. 


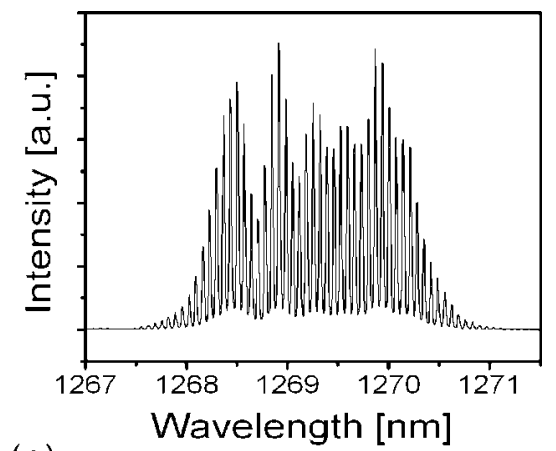

(a)

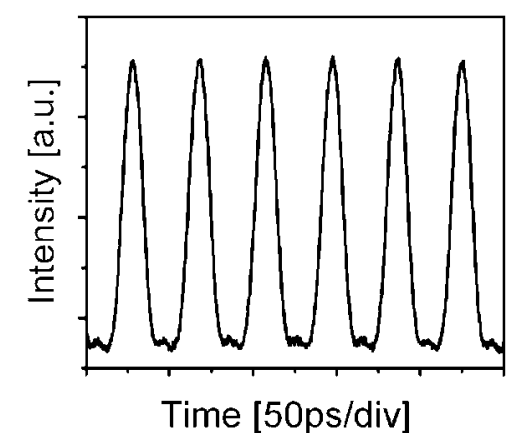

(b)

FIG. 2. (a) Optical spectrum and (b) mode-locked pulse trains.

of $\mathrm{rf}$ detuning away from the minimum noise point with respect to the supermode spur power is shown in Fig. 3(b). The supermode noise power level was less than $-120 \mathrm{dBc} / \mathrm{Hz}$, and was maintained over an $80 \mathrm{kHz}$ detuning range. Beyond this range, the contrast in optical frequency combs is reduced, while the noise spur level increases dramatically, indicating that many longitudinal mode groups are involved in the mode-locking process.

Figure 4 shows the single-sided phase noise $L(f)$ and integrated timing jitter at the optimum condition, measured with the Agilent E5505 phase noise measurement system. Two distinctive features of this QD laser are noticed. First, substantially lower phase noise $(>10 \mathrm{~dB})$ is obtained at lowfrequency offsets, as compared with QW-based lasers. ${ }^{13}$ Second, the phase noise of the supermodes is suppressed down to $-136 \mathrm{dBc} / \mathrm{Hz}$. We note that this level of supermode noise has been obtained by employing high-finesse Fabry-Pérot etalons inside laser cavities as a supermode suppression mechanism in QW-based mode-locked lasers. ${ }^{11,13}$

At low offset frequencies less than $10 \mathrm{kHz}$, the phase noise is predominantly $1 / f$, while the white noise behavior beyond $5 \mathrm{MHz}$ is considered primarily the signalspontaneous beat noise owing to optical amplification, rather than the laser output itself. The bump between $10 \mathrm{kHz}$ and $1 \mathrm{MHz}$ has been observed in both QW- and QD-based low noise lasers at the same position with a similar synthesizer, where the position of the bump and noise level coincide with the phase noise specification of the Agilent $\mathrm{rf}$ synthesizer E8247. Our analysis ${ }^{14}$ shows that the bump is a result of incomplete cancellation of the synthesizer phase noise in the local oscillator (LO) and rf port of the mixer used as a phase detector, due to a damping of the high-frequency phase noise component in the laser. The utilization of a quieter oscillator as a mode-locking frequency source is expected to lower this

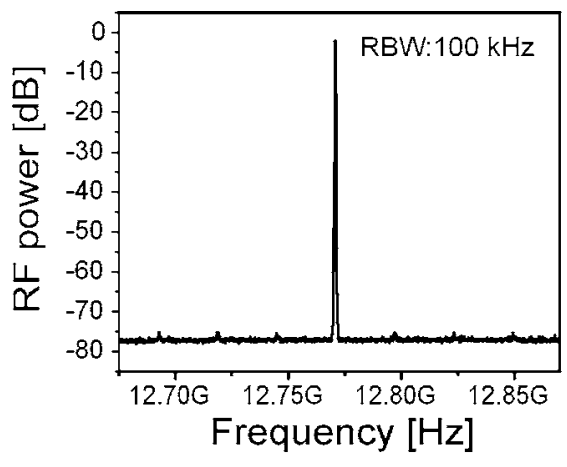

(a)

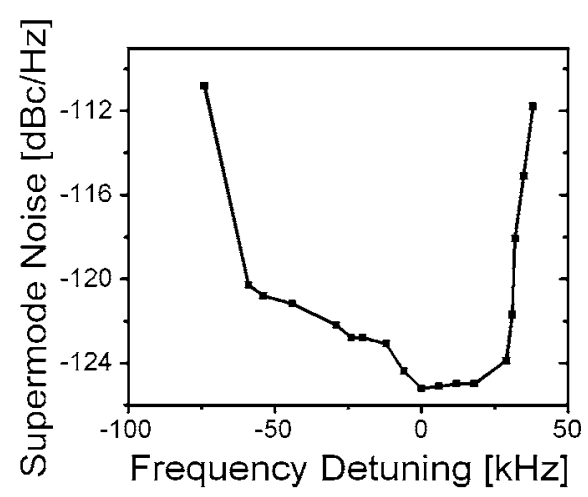

(b)

FIG. 3. (a) rf spectrum and RBW, resolution bandwidth, and (b) supermode noise level vs rf frequency detuning.

bump level and improve the measurement accuracy. The timing jitter calculated through

$$
\Delta t=\frac{1}{2 \pi f_{\bmod }} \sqrt{2 \int_{f_{\min }}^{f_{\max }} L(f) d f}
$$

gives $\sim 7.5 \mathrm{fs}$ integrating from $1 \mathrm{~Hz}$ to $10 \mathrm{MHz}$, which is the lowest residual timing jitter reported from an actively mode-locked semiconductor laser. This result shows that quantum-dot mode-locked lasers are promising as sources for ultralow noise optical pulse trains.

In summary, we studied the characteristics of an activemode-locked laser cavity based on quantum-dot SOAs. The

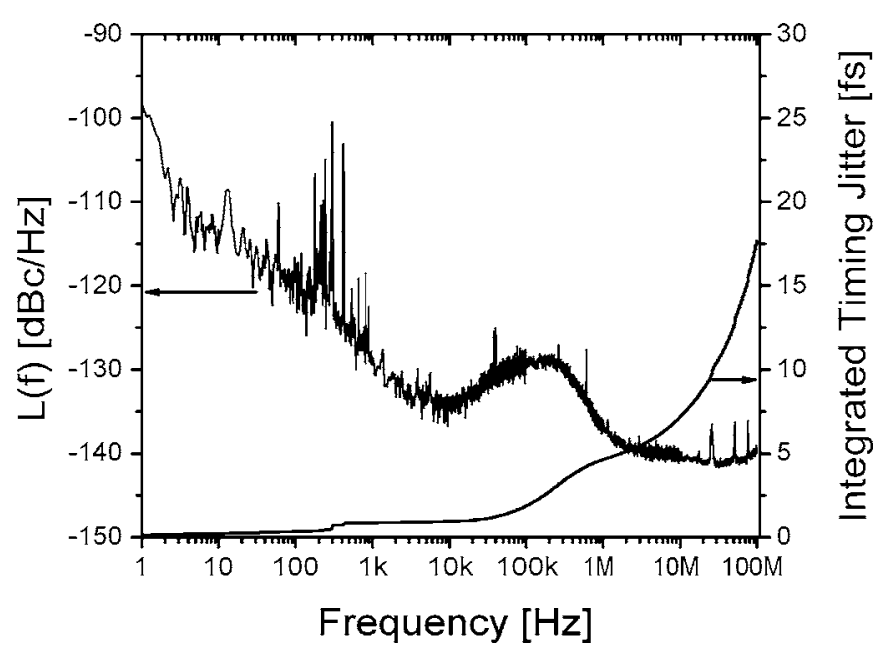

FIG. 4. Residual phase noise and integrated timing jitter. 
enhanced performance of the QD laser over conventional QW/bulk-based lasers in terms of phase noise properties was obtained. The supermode phase noise is suppressed down to $-136 \mathrm{dBc} / \mathrm{Hz}$, and stable optical combs are observed. The residual timing jitter is less than $7.5 \mathrm{fs}(1 \mathrm{~Hz}$ to $10 \mathrm{MHz})$, limited by driving synthesizer noise. This result shows that quantum-dot gain media are promising as sources for ultralow noise optical pulse generation.

${ }^{1}$ D. J. Derickson, P. A. Morton, J. E. Bowers, and R. L. Thornton, Appl. Phys. Lett. 59, 3372 (1991).

${ }^{2}$ D. J. Jones, K. W. Holman, M. Notcutt, Jun Ye, J. Chandalia, L. A. Jiang, E. P. Ippen, and H. Yokoyama, Opt. Lett. 28, 813 (2003).

${ }^{3}$ S. Gee, F. Quinlan, S. Ozharar, P. J. Delfyett, J. J. Plant, and P. W. Juodawlkis, Opt. Lett. 30, 2742 (2005).

${ }^{4}$ L. A. Jiang, M. E. Grein, E. P. Ippen, C. McNeilage, J. Searls, and H. Yokoyama, Opt. Lett. 27, 49 (2002).

${ }^{5}$ F. Rana, Rajeev J. Ram, and H. A. Haus, IEEE J. Quantum Electron.
QE-40, 41 (2004).

${ }^{6}$ F. Rana, Harry L. T. Lee, R. J. Ram, M. E. Grein, L. A. Jiang, E. P. Ippen, and H. A. Haus, J. Opt. Soc. Am. B 19, 2609 (2002).

${ }^{7}$ D. R. Hjelme and A. R. Mickelson, IEEE J. Quantum Electron. QE-28, 1594 (1992)

${ }^{8}$ T. W. Berg, and J. Mørk, Appl. Phys. Lett. 82, 3083 (2003).

${ }^{9}$ K. Komori, S. Arai, and Y. Suematsu, IEEE Photonics Technol. Lett. 3, 39 (1991).

${ }^{10}$ T. Akiyama, M. Ekawa, M. Sugawara, K. Kawaguchi, H. Sudo, H. Kuwatsuka, H. Ebe, A. Kuramata, and Y. Arakawa, in Proceedings of Optical Fiber Communication, Anaheim, CA, 6-11 March 2005 (unpublished), Paper No. OWM2.

${ }^{11}$ C. M. DePriest, T. Yilmaz, P. J. Delfyett, Jr., S. Etemad, A. Braun, and J. Abeles, Opt. Lett. 27, 719 (2002).

${ }^{12}$ M. F. Becker, D. J. Kuizenga, and A. E. Siegman, IEEE J. Quantum Electron. QE-8, 687 (1972).

${ }^{13}$ T. Yilmaz, C. M. Depriest, P. J. Delfyett, Jr., S. Etemad, A. Braun, and J. Abeles, Opt. Express 11, 1090 (2003).

${ }^{14}$ F. Quinlan and S. Gee (private communication). 